\title{
Incidence of filamentosus fungi in sputum of patients affected by cystic fibrosis
}

\author{
Claudia Cutrini', Annamaria Calvo', Rolando Gagliardini², Natalia Cirilli², Esther Manso' \\ I SOS Microbiologia-Ospedali Riuniti di Ancona \\ 2 Centro Regionale Fibrosi Cistica, SOD Pediatria, POAS “G. Salesi”-Ospedali Riuniti di Ancona
}

Key Words: Cystic Fibrosis, Filamentous fungi, Exophiala dermatitidis, Scedosporium apiospermium, Allergic bronchopulmonary aspergillosis, Invasive pulmonary infections

Incidenza dei funghi filamentosi in espettorati di pazienti con fibrosi cistica

\section{SUMMARY}

Introduction. Cystic fibrosis (CF) is an autosomal recessive genetic disorder caused by a mutation of the gene encoding the CF protein CFTR. Patients with CF are frequently affected by respiratory infections due to specific pathogens. The role of filamentous fungi is well established in the case of allergic bronchopulmonary aspergillosis and invasive pulmonary infections in lung transplant patients, but their involvement in lung diseases outside of these cases remain to be clarified. The aim of our study was to monitor the impact of filamentous fungi in patients with CF.

Methods. In the years 2007 and 2008 and during the first half of 2009 a total of 1046 samples of sputum collected from CF patients were analyzed. Of these 236 cases were positive for fungi.

Results. Filamentous fungi were isolated from 44 patients, including 29 females and 15 males. The fungi were identified as Aspergillus fumigatus $(70.04 \%$ of the cases), A. terreus (2.4\%),A.flavus (II.4\%), Scedosporium apiospermum (3.8\%), Penicillium spp (0.84\%), Aspergillus spp. (I.26\%) and Exophiala dermatitidis (0.24\%).

Conclusions. Based on the isolates, out of 44 cases the duration in time of colonization by filamentous fungi was constant with the same species in II patients, intermittent with the same or occasionally another species in 14 patients and present for no more than one semester in the remaining 19 cases.

La Fibrosi Cistica (FC) è una malattia autosomica recessiva causata dalla mutazione del gene che codifica per la proteina CFTR. I pazienti FC sono spesso soggetti ad infezioni respiratorie causate da patogeni specifici (Pseudomonas aeruginosa, Staphylococcus aureus, B. cepacia complex) per quali sono state già validate terapie antibiotiche opportune. Con il miglioramento delle tecniche di isolamento per la determinazione dei miceti si è riscontrato nell'escreato anche la presenza di miceti filamentosi, quali A. fumigatus, A. terreus, A. flavus e due nuovi patogeni emergenti S. apiospermum ed E. dermatitidis. Sebbene il ruolo di alcuni miceti sia ancora da chiarire, sembra che la colonizzazione fungina agisca simultaneamente con la colonizzazione batterica. A. fumigatus è responsabile di diverse malattie come: asma, bronchite, aspergilloma, aspergillosi broncopolmonare allergica (ABPA) e non per ultima l'aspergillosi polmonare invasiva (IA) ampiamente temuta dai pazienti trapiantatati. Una colonizzazione cronica con forme cliniche simili a quelle studiate in A. fumigatus, ABPA e IA, è stata osservata anche con A. terreus. S. apiospermum ha acquisito importanza nel tempo poiché contribuisce alla reazione infiammatoria ed al progressivo deterioramento delle funzioni polmonari. La sua presenza è una controindicazione per il trapianto di polmone per la possibilità di micosi cerebrale. S. apiospermum è generalmente responsabile di una colonizzazione cronica dell'albero respiratorio ma raramente è associata a segni clinici. $E$.

\section{COLONIZZAZIONE FUNGINEA CRONICA}

(totale nei tre anni )

TIPO DI FUNGO $\quad$ SINGOLO $\quad$ ASSOCIATI

\begin{tabular}{lcc}
\hline A. fumigatus & 112 & 17 \\
\hline A. flavus & - & 12 \\
\hline A. terreus & 16 & 5 \\
\hline Penicillium spp. & 1 & - \\
\hline S. apiospermium & 9 & - \\
\hline Aspergillus spp. & 2 & - \\
\hline Exophiala dermatitidis & $\mathrm{I}$ & -
\end{tabular}

dermatitidis, lievito nero a $37^{\circ} \mathrm{C}$ e fungo filamentoso a temperatura ambiente, è considerato un biomarker di malattia CFTR - correlata in pazienti con bronchiettasie.

Scopo di questo studio è stato quello di verificare l'incidenza dei miceti filamentosi isolati da pazienti affetti da FC nel tempo e correlare la presenza e la persistenza dei miceti filamentosi con l'andamento clinico.

Sono stati analizzati gli espettorati di 106 pazienti FC nel biennio 2007-08 e nel primo semestre del 2009 per un totale di 1037 campioni. Gli espettorati sono stati seminati su piastre di agar Sabouraud + cloramfenicolo (CAF) alla concentrazione di $25 \mathrm{mg} / \mathrm{L}$, incubate per 2 over-night a $35^{\circ}+2^{\circ} \mathrm{C}$ e per i successivi 5 giorni a temperatura ambiente.

L'identificazione delle colonie di funghi filamentosi è stata eseguita mediante: osservazione dell'aspetto macroscopico e riconoscimento microscopico.

Sono stati isolati funghi filamentosi in 43 pazienti di cui 28 femmine e 15 maschi. In 14 pazienti la colonizzazione è risultata intermittente con la stessa specie o saltuaria con un'altra specie. In 13 pazienti sono state isolate più di una specie $:$ A. fumigatus + A. flavus $=6$ A. fumigatus $+A$. terreus $=4 \mathrm{~A}$. terreus $+\mathrm{A}$. flavus $=2 \mathrm{~A}$. fumigatus $+\mathrm{S}$. apiospermum $=1$

La colonizzazione cronica con la stessa specie fungina è stata osservata in 19 pazienti con A. fumigatus, in 3 con A. terreus, in 3 con A. flavus e nei 2 con S. apiospermum.

\section{COLONIZZAZIONE FUNGINEA SPORADICA} (totale nei tre anni ) TIPO DI FUNGO

\begin{tabular}{lcc} 
TIPO DI FUNGO & SINGOLO & ASSOCIATI \\
\hline A. fumigatus & 26 & 4 \\
\hline A. flavus & $1 \mathrm{I}$ & 3 \\
\hline A. terreus & 2 & 3 \\
\hline Penicillium spp. & $\mathrm{I}$ & - \\
\hline S. apiospermium & - & - \\
\hline Aspergillus spp. & $\mathrm{I}$ & - \\
\hline Exophiala dermatitidis & - & - \\
\hline
\end{tabular}

N.B. - Per colonizzazione fungina cronica si intende che il paziente è stato colonizzato dallo stesso fungo per almeno un semestre. Per colonizzazione fungina sporadica si intende che il fungo si è isolato per meno di sei mesi.

\section{Corresponding author: Claudia Cutrini,}

Ospedali Riuniti Lancisi,SOS Microbiologia - Via Conca I - 60 I00, Ancona, Italia. - Tel: +39 07I.5964250

E-mail: claudia.cutrini@yahoo.it 
Dei 1037 campioni analizzati 206 sono i campioni di pazienti risultati positivi per funghi filamentosi.

Di questi campioni 22 presentano due specie fungine associate (tabella).

Gli Aspergilli sono i miceti filamentosi riscontrati con mag- giore frequenza nell'albero respiratorio. In particolare sono stati isolati nei 43 pazienti:A. fumigatus $(72 \%)$, A. terreus (23\%), A. flavus (23\%). Isolati con minore frequenza i patogeni emergenti come S. apiospermum (4\%) ed E. dermatitidis $(2 \%)$.

\section{SPECIE FUNGINE IDENTIFICATE}

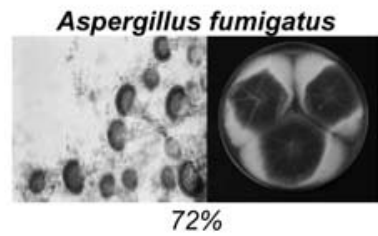

Scedosporium apiospermum

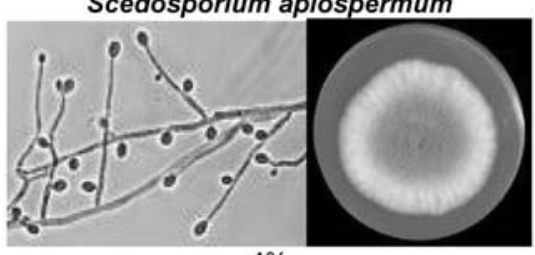

$4 \%$

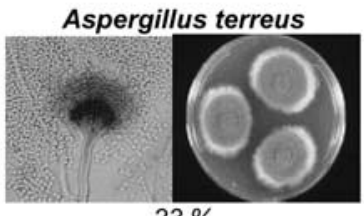

$23 \%$

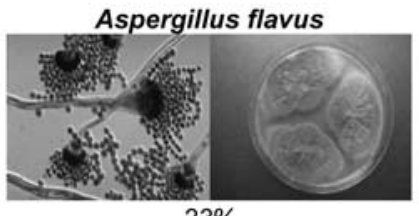

$23 \%$

\section{Isolamento dei funghi nei campioni negli anni}

$\begin{array}{lll}\text { = Asp.Fumigatus } & \text {-Asp.flavus } & \text { - Asp.terreus } \\ \text { = Scedosporium } & \text { - Penicillium } & =\text { Asp.spp }\end{array}$

Exophiala dermatitidis

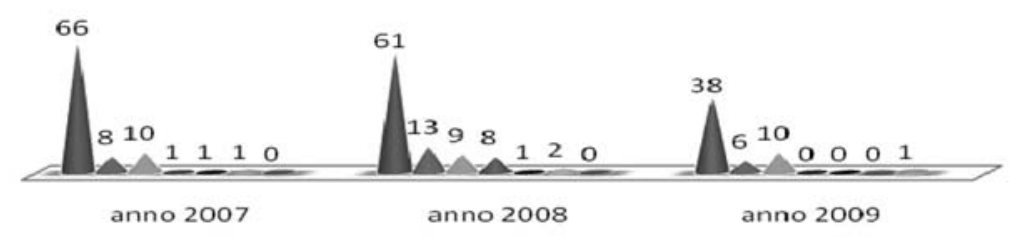

\section{L'ANDAMENTO CLINICO}

Nel periodo considerato tre pazienti colonizzati cronicamente da A. fumigatus (in due casi associato a A. terreus) hanno sviluppato ABPA e sono stati trattati con un antifungino (itraconazolo o voriconazolo) e/o corticosteroidi.

Altri quattro pazienti, in assenza di ABPA (uno dei quali con pregressa $A B P A$ risolta) sono stati trattati con antifungino (itraconazolo o voriconazolo) per ripetuta presenza nell'escreato di funghi filamentosi e peggioramento della funzione respiratoria dovuta probabilmente alla sinergia della colonizzazione fungina con quella batterica; in tutti i 4 casi era stato isolato $A$. fumigatus, associato in 1 caso con $A$. terreus e in 1 con $S$. apiospermum.

I risultati delle terapie sono ancora oggetto di studio .

A. fumigatus è il micete filamentoso che colonizza più frequentemente l'albero respiratorio dei pazienti con $\mathrm{FC}$, seguito da A. terreus. A. fumigatus è stato isolato nei 7 pazienti sottoposti a terapia antifungina. Tra i 7 pazienti, 3 erano anche colonizzati con A. terreus. Tre dei 7 pazienti presentavano un

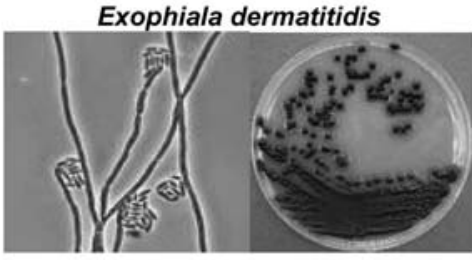

$1 \%$

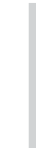

